\title{
Analysis and Improvement of SNR in FBG Sensing System
}

\author{
Delong $\mathrm{KONG}^{1}$, Jun $\mathrm{CHANG}^{1}$, Peijun $\mathrm{GONG}^{1}$, Yongning $\mathrm{LIU}^{1}$, Boning $\mathrm{SUN}^{1}$, \\ Xiangzhi $\mathrm{LIU}^{2}$, Pengpeng $\mathrm{WANG}^{1}$, Zongliang $\mathrm{WANG}^{1}$, Weijie WANG ${ }^{1}$, and Yan $\mathrm{ZHANG}^{1}$ \\ ${ }^{1}$ School of Information Science and Engineering and Shandong Provincial Key Laboratory of Laser Technology and \\ Application, Shandong University, Jinan, 250100, China \\ ${ }^{2}$ Institute of Automation Shandong Academy of Sciences, Jinan Shandong 250014, China \\ *Corresponding author: Jun CHANGＥ-mail: changjun@sdu.edu.cn
}

\begin{abstract}
The improvement of the signal to noise ratio (SNR) has significant meaning to the fiber Bragg grating (FBG) sensing system. The source of the noise as well as the signal attenuation of the FBG sensing system is analyzed. It is found that optical noise caused by the optical return loss (ORL) is the main source of noises in the system, and the coupler is the main source of attenuation of the signal. The cause of the ORL in fiber-optic elements (such as jumper cables connector and fiber end) is presented. In addition, suggestions to optimize the fiber optical sensing network in order to improve the SNR are presented. Methods to suppress noises caused by the fiber end interfaces of FBGs, including using index-matching fluid, bending fiber pigtails in the way mentioned in this paper and cleaving the slant angle of the fiber interfaces to be $8^{\circ}$, all contribute to the optimized SNR. Besides, the thermo-weld method is suggested to be used for both parallel and serial FBG setups to provide a low insertion loss. The results would be a useful engineering tool to design the high SNR optical sensing system.
\end{abstract}

Keywords: SNR, return noise, return loss, fiber Bragg grating, reflection of fiber end interface, bending loss

\section{Introduction}

Wavelength demodulation is the crucial part of the fiber Bragg grating (FBG) sensing system, by which the information carried by an FBG can be discriminated accurately in the form of the wavelength change [1].

Noise is a so important factor affecting the accuracy of FBG spectrum analyzers [2] that the information that the FBG carries can not be demodulated properly once the intensity of the noise is greater than that of the signal. The noise of the FBG sensing system contains the thermal noise coming from the demodulation instrument itself [3] and the optical noise coming from the system. Since the thermal noise is as little as $-70 \mathrm{~dB}$ according to our experiments, so it can be ignored when compared with the optical noise. The main components of the optical noise are formed by return noise and Rayleigh scattering noise [4], and to our knowledge, the noise caused by the Rayleigh scattering is about $55 \mathrm{~dB}$ lower than that of the input light [5]. So the Rayleigh scattering noise is weak and not discussed in this paper.

This paper shows clearly the main sources of the return noise based on our experiments and how to reduce the return noise and increase the signal to noise ratio (SNR). The good SNR is the premise of accurate wavelength demodulation, and an optical device can be used properly to obtain a good SNR

Received: 14 November 2011 / Revised version: 10 February 2012

C The Author(s) 2012.This article is published with open access at Springerlink.com 
with the minimum cost by the measures showed in this paper.

\section{Theory}

(1) SNR is a measure that compares the level of the desired signal to the level of the background noise. It is defined as

$$
S N R(\mathrm{~dB})=10 \lg \left(\frac{P_{\text {signal }}(\mathrm{mW})}{P_{\text {noise }}(\mathrm{mW})}\right)
$$

where $P_{\text {signal }}$ and $P_{\text {noise }}$ are the power values of the signal and noise with the units in $\mathrm{mW}$, respectively [6].

(2) Optical return loss (ORL) is a measure of the power reflected from a discontinuity relative to the power incident upon it. It can be expressed as

$$
\operatorname{ORL}(\mathrm{dB})=10 \lg \left\{\frac{P_{\mathrm{i}}(\mathrm{mW})}{P_{\mathrm{r}}(\mathrm{mW})}\right\}
$$

where $O R L$ is the return loss of optical devices in decibel $(\mathrm{dB})$ unit, $P_{\mathrm{i}}$ is the power of the incident light, and $P_{\mathrm{r}}$ is the power of the reflected light.

(3) Fiber end reflection theory:

In optical fiber devices, the light reflected from the fiber end strengthens the noise. The reflectance of the fiber end shows as follows [7-9]:

$$
\begin{aligned}
R= & 10 \lg \left\{\frac{\left(n_{1}-n_{0}\right)^{2}}{\left(n_{1}+n_{0}\right)^{2}} \cdot \frac{2 \cos 2 \theta}{1+\cos ^{2} 2 \theta} \times\right. \\
& \left.\exp \left[-\frac{(2 \theta)^{2}}{2\left(1+\cos ^{2} 2 \theta\right)}\left(n_{1} k \omega_{0}\right)^{2}\right]\right\}
\end{aligned}
$$

where $n_{1}$ is the refractive index of the fiber core, $n_{0}$ is the refractive index of the medium in front of the fiber end, $\theta$ is the slant angle of the fiber end, $\kappa=2 \pi / \lambda$ is the propagation constant (in our system, $\lambda$ ranges from $1525 \mathrm{~nm}$ to $1565 \mathrm{~nm}$ ), and $\omega_{0}$ is the waist radius, which is equal to the mode field diameter.

For the fiber (SMF-28E, CORNING) used in our experiments, $n_{1}$ is equal to 1.4682 , and $\omega_{0}$ is equal to $5.2 \mu \mathrm{m}$.
(4) Bending losses of single-mode fibers:

For step-index single-mode fibers (SMF), we assume $r$ as the radius of the curvature, so the bending loss per nanometer is given in (4).

$$
\alpha=A_{c} r^{-\frac{1}{2}} \exp (-U r)
$$

where $U \approx 0.705 \frac{\Delta^{\frac{3}{2}}}{\lambda}\left(2.748-0.996 \frac{\lambda}{\lambda_{c f}}\right)^{3} \quad, \quad A_{c} \approx$ $30 \Delta^{\frac{1}{4}} \lambda^{-\frac{1}{2}}\left(\frac{\lambda_{c f}}{\lambda}\right)^{\frac{3}{2}}$, and $\Delta=\left(n_{1}-n_{2}\right) / n_{2} \quad[10]$

The cutoff wavelength, $N A$ and the refractive index of the fiber core for SMF-28E are $1260 \mathrm{~nm}$, 0.14 and 1.4682 , respectively. Based on these three parameters, we calculate $n_{2}=1.4615$ from $n_{1}=1.4682$ and $N A=0.14$.

As shown in Fig. 1, when the fiber is twined around the cylinder for 10 times in the radius of $5 \mathrm{~mm}$, the bending loss is calculated to be $63 \mathrm{~dB}$ which can effectively suppress the noise.

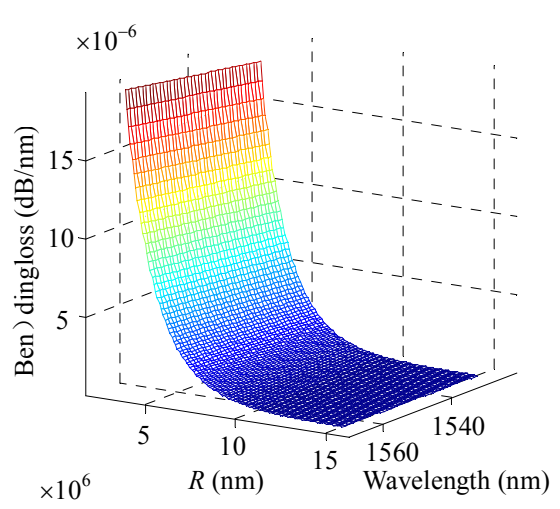

Fig. 1 Dependence of bending loss on the bending radius and wavelength.

\section{Experimental setup}

The schematic of our experimental setup in Sections 4.1 and 4.2 is shown in Fig. 2. The light emitted by the amplified spontaneous emission (ASE) source, goes through a 50/50 fiber coupler and then enters a $1 \times 8$ splitter, where the light is split into 8 FBG sensors through fiber jumpers and connectors. The signal reflected by the FBGs goes 
along the optical path and reaches the FBG spectrum analyzer which is connected to the other input port of the fiber coupler. The power value measured with the optical power meter at the output port of the coupler is $12.35 \mathrm{dBm}$, and it keeps the same in following experiments and analyses.

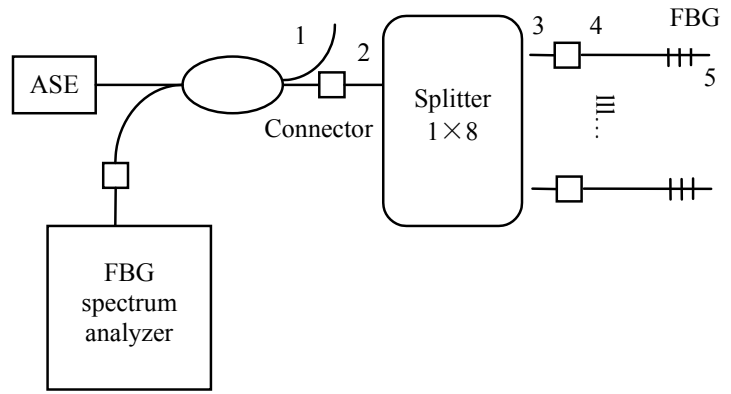

Fig. 2 Schematic of the experimental setup with parallelly connected FBG arrays.

The experiments in Section 4.3 use the same light source as experiments in Sections 4.1 and 4.2. The FBGs are connected to each other in a string with a fiber connector, and the fiber pigtail of the last FBG is cleaved with an $8^{\circ}$ angle as shown in Fig. 3.

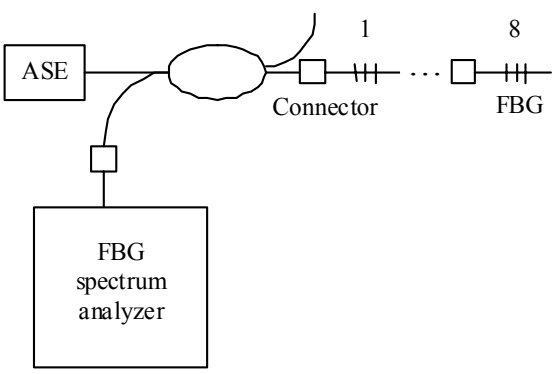

Fig. 3 Schematic of the experimental setup with serially connected FBG arrays.

\section{Experiments, results and analysis}

\subsection{Determining factors of the power of the signal and noise in the parallel connection}

In engineering applications, FBG sensors are usually connected parallelly or serially. The parallel connection is widely used because the optical path is convenient to be designed and fixed in optical systems. The optical path of the parallel connection was tested and analyzed at first, and the factors affecting the SNR were recognized in this paper.
Then, according to the contrastive tests of the SNR between the parallel and serial connections, we decided which kind of connections was easier to acquire a high SNR.

The power of the signal light is affected by insertion loss and the reflectance of the FBG Moreover, the Rayleigh scattering noise, the return noise, and the thermal noise from the device are the main sources of the noises [11, 12]. We recognized the determining factors of the signal power and noise power with a modified version of the setup shown in Fig. 2. In our setup, one output of the splitter was connected to a reflector and the others were connected to air instead. Thus, the highest signal power was what the FBG spectrum analyzer received when the reflectance of the $\mathrm{FBG}$ was equivalent to $100 \%$.

Then the fiber pigtail of the output end of the coupler was twined around the cylinder for 10 times in the radius of $5 \mathrm{~mm}$. And in the following parts of this paper, fiber bending was introduced with this method. The bending loss of the fiber around the cylinder was estimated to be $63 \mathrm{~dB}$ at least. Consequently, the light reflected by the end of the ferrule reduced so sharply that it could be ignored when compared to the return loss caused by the coupler itself. The fiber would be dealt with in this way. The FBG spectrum analyzer showed that the noise of the system was very small, and almost all the noise was caused by the coupler itself. So the value of the maximum signal and the minimum noise could be found out.

As Fig. 4 illustrates, Curve $\mathrm{D}$ shows the maximum value of the signal, and Curve $B$ shows the minimum value of the noise.

The signal reduces by $6 \mathrm{~dB}$ because of $3-\mathrm{dB}$ coupler in the experimental setup mentioned above. But when the coupler is replaced by a fiber circulator, the reduction can decrease to about $1 \mathrm{~dB}$ [13]. Besides, the splitter can attenuate the reflected signal obviously. The more ports the splitter has, the higher the attenuation is. 


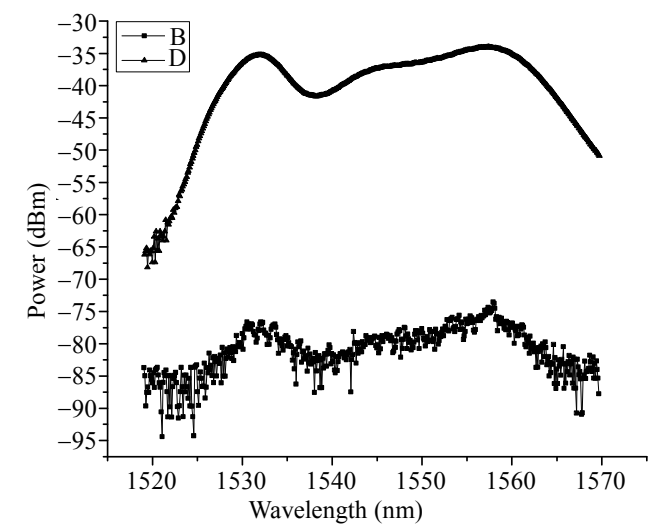

Fig. 4 Power of the maximum signal and the minimum noise in the FBG sensing system in the parallel setup.

The SNR of the system becomes higher when the insertion loss of the signal reduces and the return loss of the optical devices increases. An optical device with good quality works with low insertion loss and high return to provide a high SNR [14-16].

Because the power of the noise is much easier to be affected by the fiber end face than that of the signal, the noise is the dominant factor for the SNR in the parallel connection setup.

\subsection{Determining factors of the return noise}

\subsubsection{Influences of the optical devices in the system on the total return noise}

In order to identify the influences of the optical devices from different parts of the system on the total return noise, the fibers at Positions 1, 2, 4 and 5 in Fig. 2 were bent sequentially for 4 separate measurements. The measured return noises by the FBG spectrum analyzer are shown as Curves D, F, H and B in Fig. 5.

As seen in Fig. 5, the return noise accumulates as the light travels further. And the more devices the system has, the higher return noise the FBG spectrum analyzer can receive.

The return loss of the fiber optical system in Fig. 2 is usually caused by the coupler, the connector behind the coupler, the splitter, the connector behind the splitter, and the fiber end of the FBG. In Fig.5, Curves D, F, H, and B represent, respectively

$$
\begin{aligned}
& P_{D}(\mathrm{dBm})=P_{\text {coupler }} \quad, \quad P_{F}(\mathrm{dBm})=10 \lg \left(10^{\frac{P_{\text {coupler }}}{10}}+\right. \\
& \left.10^{\frac{P_{\text {commector }}}{10}}\right) \quad, \quad P_{H}(\mathrm{dBm})=10 \lg \left(10^{\frac{P_{\text {coupler }}}{10}}+10^{\frac{P_{\text {comector }}}{10}}+\right. \\
& \left.10^{\frac{P_{\text {spititer }}}{10}}+\sum_{i=1}^{8} 10^{\frac{P_{\text {compector }}}{10}}\right) \text { and } P_{B}(\mathrm{dBm})=10 \lg \left(10^{\frac{P_{\text {coupler }}}{10}}\right. \\
& \left.+10^{\frac{P_{\text {commector }}}{10}}+10^{\frac{P_{\text {splititer }}}{10}}+\sum_{i=1}^{8} 10^{\frac{P_{\text {commector }}}{10}}+\sum_{i=1}^{8} 10^{\frac{P_{\text {endface }}}{10}}\right)[17]
\end{aligned}
$$

where $P$ is the power of the return loss in $\mathrm{dBm}$ unit.

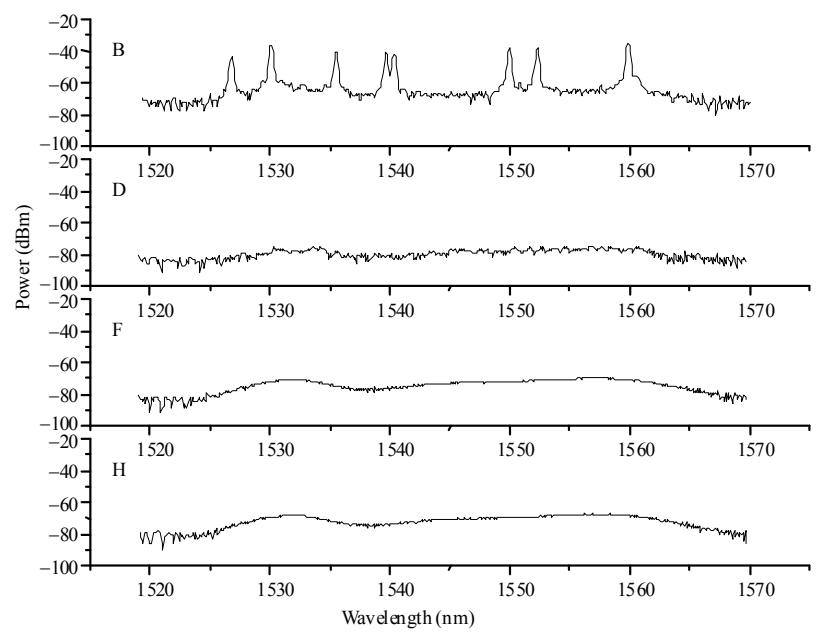

Fig. 5 Spectra detected by the FBG spectrum analyzer when the fibers at Positions 1, 2, 4 and 5 were bent sequentially.

FBT and PLC are two types of splitters which have been widely used in practical applications. Both of them are connected to other devices with fiber connectors. The connectors near the input end and the output end of the splitter and the splitter itself can increase the return noise. Generally speaking, the return loss of a connector, $O R L_{\text {connector, }}$ is supposed to be an estimated value, about $50 \mathrm{~dB}$ to $60 \mathrm{~dB} . P_{\text {in }}$ and $P_{\text {out }}$ are the input power and output power of the splitter in $\mathrm{dBm}$ unit, respectively. $\Sigma X$ stands for the loss of the light in $\mathrm{dB}$ unit when it propagates from the input of the splitter to the FBG spectrum analyzer. So the return noise caused by the input end connector of the splitter is $P_{\text {in }}-O R L_{\text {connector }}$ $\Sigma X$, and for the output end connector, it is

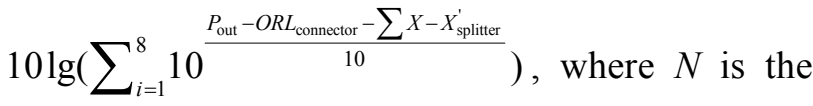
number of the output ports of the splitter, $X_{\text {splitter }}$ and $X_{\text {splitter }}^{\prime}$ are the insertion losses of the splitter in 
forward direction and reverse direction separately, and to our knowledge, $X_{\text {splitter }}$ is equal to $X_{\text {splitter. }}^{\prime}$. Theoretically, $X_{\text {splitter }}$ is equal to $-10 \lg (1 / N)$. And the measured insertion loss of splitters in practice corresponds well with the theoretical value due to the ripe manufacturing process. So the relationship between the $X_{\text {splitter }}$ and the noise to noise ratio (NNR) defined as the return noise ratio caused by the output end connector of the splitter to the input one, can be expressed as

$$
N N R(\mathrm{~dB})=10 \lg \left(N \cdot 10^{-\frac{2 X_{\text {spliter }}}{10}}\right) .
$$

Substituting $X_{\text {splitter }}=10 \lg (1 / N)$ into the above formula yields that $N N R(\mathrm{~dB})=10 \lg (1 / N)$. Figure 6 depicts how $N N R(\mathrm{~dB})$ changes with $N$ $(N=2,4,8,16,32,64)$.

The splitter used in our experiments has 8 output ports. According to Fig. 6, the return noise caused by the output end connector is about $9.03 \mathrm{dBm}$ lower than that caused by the input end connector. As seen in Fig. 6, the return noise caused by the connector of the input end of the splitter is much larger than that caused by the connector of the output end.

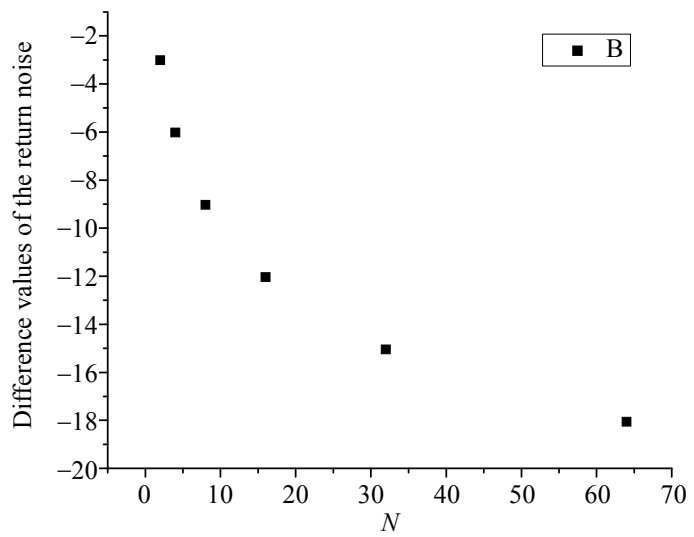

Fig. 6 Simulation of the relationship between the $N N R$ and $N$ $(N=2,4,8,16,32,64)$.

As a result, the part of Curve $\mathrm{H}$ higher than Curve $\mathrm{F}$ is caused by the return noise of the splitter itself. Meanwhile, the return noise reflected by the fiber end of FBGs induces the higher part of Curve B than Curve H.

\subsubsection{Influences of the fiber end face situations on the total return noise}

Optical devices in FBG sensing systems can cause the return noise. [18] The FBG sensors are usually fixed in the end of the optical path of the parallel connection setup, and each end face of the FBG can reflect a portion of the light. In practice, the high dependence of the SNR on the situation of the fiber end face can not be ignored.

For the optical setup shown in Fig. 2, the spectrum of the return noise received by the FBG spectrum analyzer when the fibers at Position 4 were bent with the above mentioned method is illustrated as Curve $\mathrm{R}$ in Fig. 7. We disbranched all the jumpers connected to the 8 output ports of the splitter, and the slant angle of the fiber end of FBGs was cleaved to be $0^{\circ}$. Then each jumper together with the FBG was connected to its original port of the splitter one by one, and the resultant spectrum was measured by the FBG spectrum analyzer shown in Fig. 7. The experiments in Section 4.2.2 were conducted in the same way.

In Fig. 7, Curve $\mathrm{B}$ is corresponding to the reflection spectrum when merely the first port of the splitter is connected to the FBG. It is obvious that Curve $\mathrm{R}$ is so lower than Curve $\mathrm{B}$ that it can be ignored. The return noise caused by the fiber end face is expressed as $P_{\text {noise }}=P_{\text {in }}+R-\Sigma X$, where $P_{\text {in }}$ is the incoming power at the fiber end. Because of the minor insertion loss of the connectors and FBGs, the spectrum detected by the FBG spectrum analyzer at the output port of the splitter is considered to be $P_{\text {in }}$. $R$ is the reflectance at the fiber end interface in $\mathrm{dB}$ unit. The slant angle of the fiber end is cleaved to be $0^{\circ}$. According to (3), $R$ almost keeps constant $(R \approx-14.44 \mathrm{~dB})$ for the wavelengths $(1525 \mathrm{~nm}$ to $1565 \mathrm{~nm}$ ) used in our system. $\Sigma X$ stands for the loss of the light in $\mathrm{dB}$ unit when it propagates from the input of the splitter to the FBG spectrum analyzer. According to the empirical value given by the FBG spectrum analyzer, $\Sigma X$ is about $13 \mathrm{~dB}$. Figure 8 
shows the theoretical and measured spectra when merely the first port of the splitter is connected to the FBG.
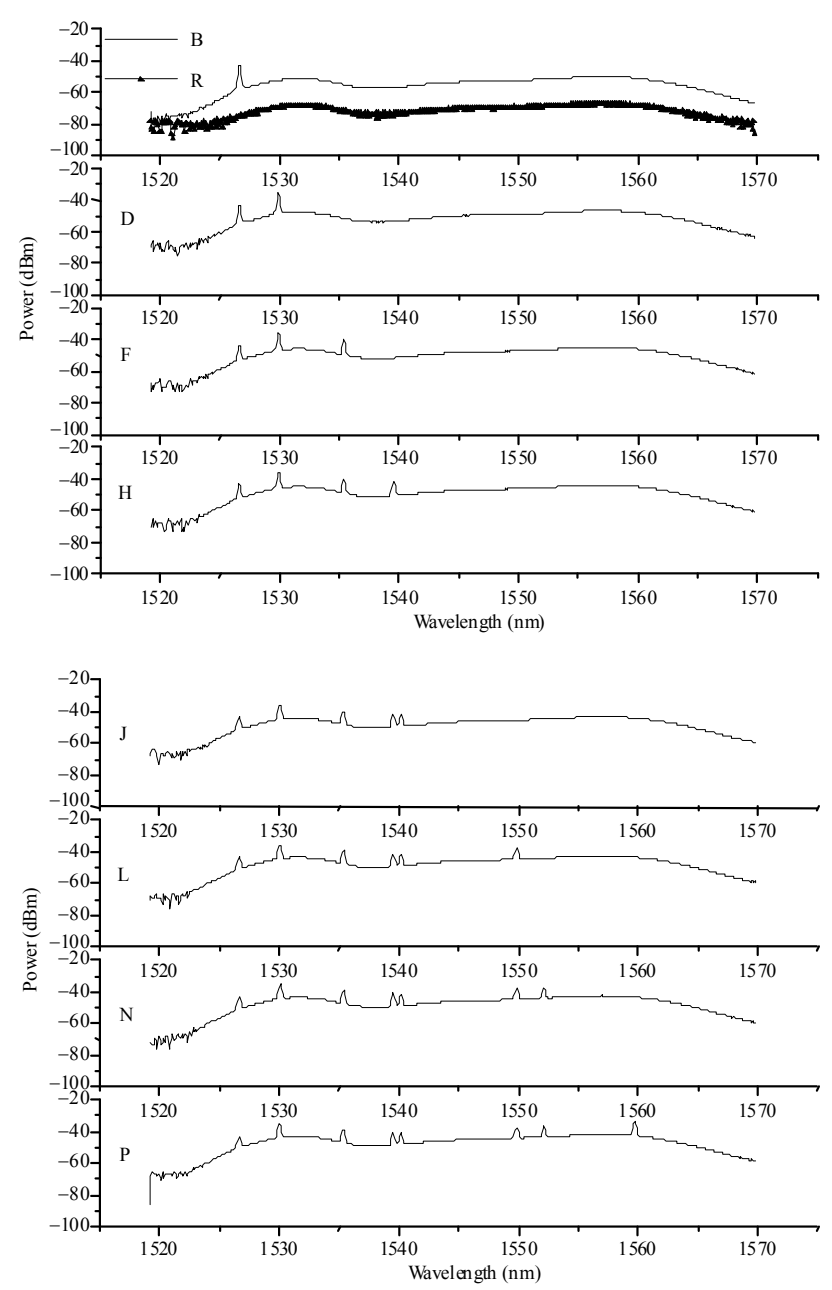

Fig. 7 Spectra of the return noise detected by the FBG spectrum analyzer when the fibers at Position 4 were bent (Curve B) and when each jumper together with FBG was connected to its original port of the splitter one by one (Curves D-R).

As shown in Fig. 8, the theoretical simulation is in good agreement with the measured spectrum. Because of the same optical setup connected to the output port of the splitter and the same fiber end interface, the return noise induced by each port of the splitter is almost the same. So when the number of the splitter ports increases to 8 , the simulation is reasonable, and the simulation fits very well with the measurement.

For the optical setup shown in Fig. 2, the slant angle of the fiber end of FBGs is cleaved to be $0^{\circ}$, and pure water $(n=1.333)$ is used as the index-matching fluid to minimize the reflection at the FBG fiber end interface in this experiment. The resultant spectra in Fig. 9 are measured by the FBG spectrum analyzer.
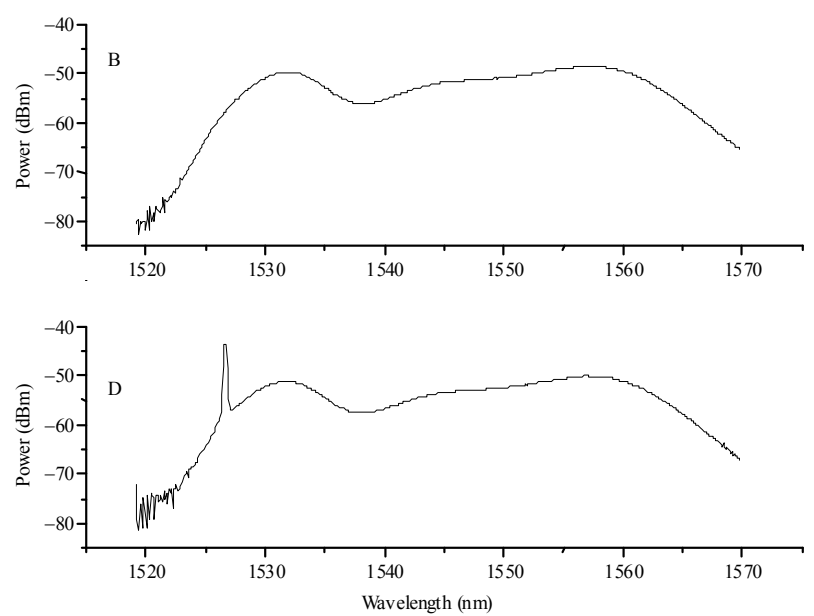

Fig. 8 Comparison of the theoretical and measured spectra when merely the first port of the splitter is connected to the FBG with the slant angle of $0^{\circ}$.

In Fig. 9, the spectrum of the return noise caused by the components before Position 4 is illustrated as Curve B. Curve D is corresponding to the reflection spectrum when merely the first port of the splitter is connected to the FBG. It is obvious that Curve B is so low (lower than Curve D) that it can be ignored. The reflectance at the fiber end interface in this experiment is $-26.35 \mathrm{~dB}$ according to (3). The return noise caused by the components along the optical path to Position 5 when merely the first port of the splitter is connected to the FBG is shown in Fig. 10 according to the analyses mentioned above.

As shown in Fig. 10, the theoretical simulation (Curve B) is in good agreement with the measured spectrum (Curve D).

According to the analyses above, the return noise is mainly caused by the reflection of the fiber end interface of FBGs. The measures in the following experiments are carried out in order to improve the SNR. 

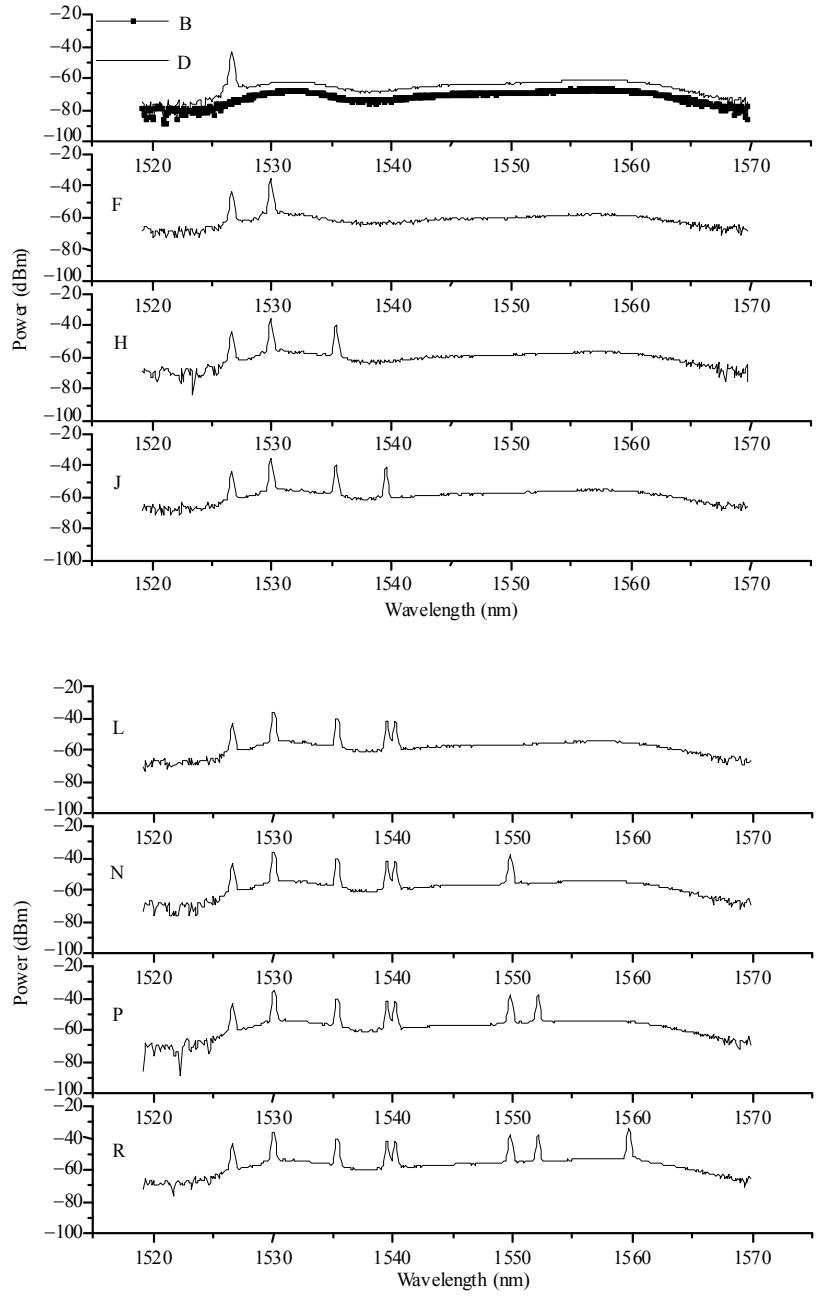

Fig. 9 Spectra of the return noise detected by the FBG spectrum analyzer when the fibers at Position 4 are bent (Curve B) and when each jumper together with the FBG is connected to its original port of the splitter one by one (Curves D-R).

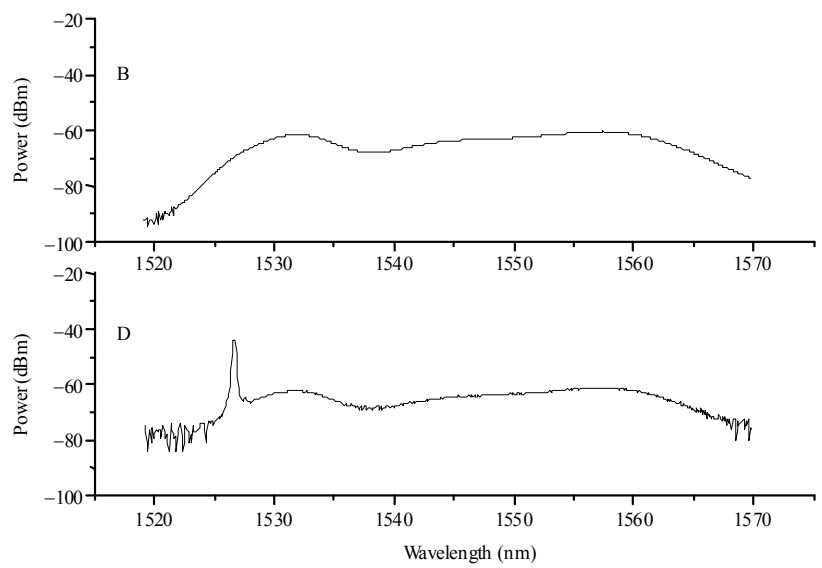

Fig. 10 Comparison of the theoretical and measured spectra when merely the first port of the splitter was connected to the FBG with the index-matching fluid.
For the optical setup shown in Fig. 2, the experiment is conducted when the fibers at Position 4 are bent with the above mentioned method. Figure 11 shows the measured spectra by the FBG spectrum analyzer.

According to (5), the bending loss of the SMF can effectively suppress the noise caused by the fiber end interface. Therefore, the return noise of the system increases inconspicuously with an increase in the number of the FBGs, and there is no significant deterioration of the SNR.
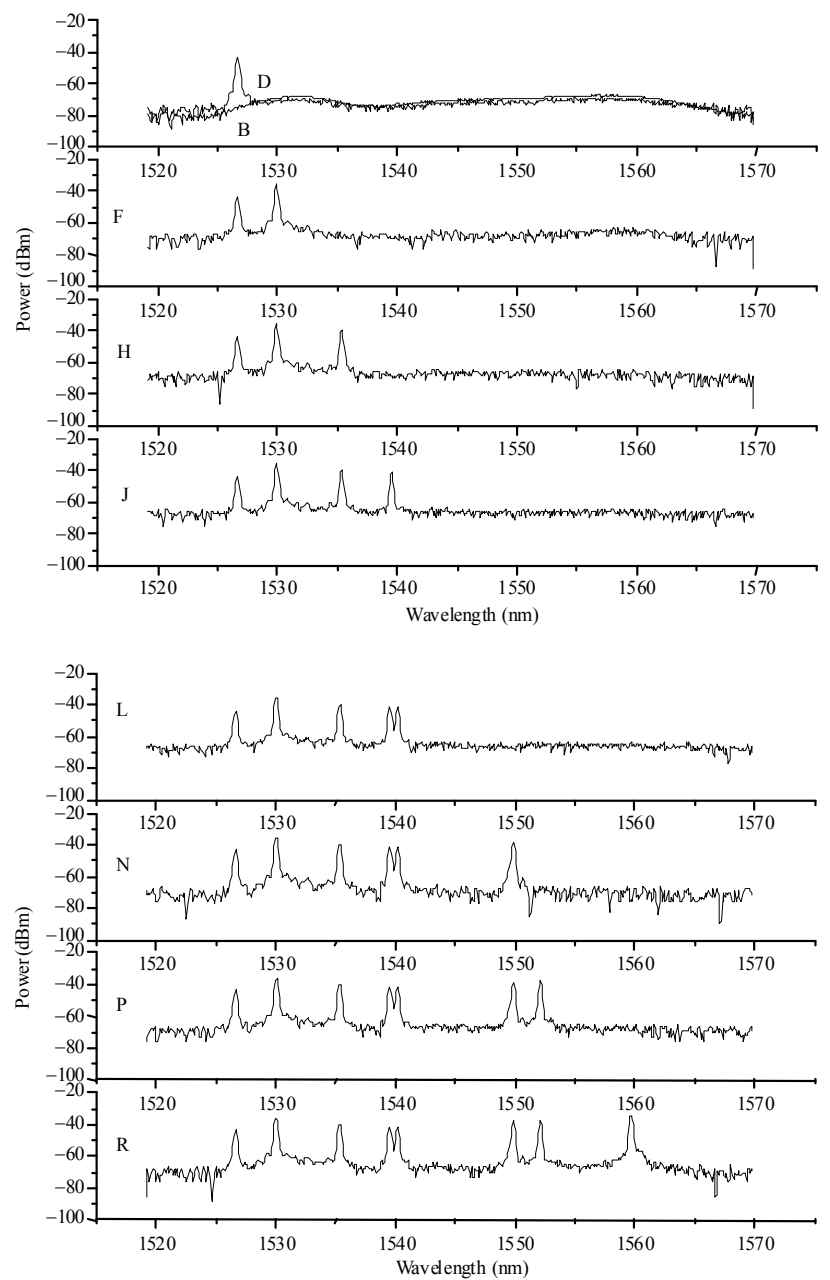

Fig. 11 Spectra of the return noise detected by the FBG spectrum analyzer when the fibers at Position 4 are bent (Curve B) and when each jumper together with the FBG is connected to its original port of the splitter one by one (Curves D-R).

For the optical setup shown in Fig. 4, the slant 
angle of the fiber end of FBGs is cleaved to be $8^{\circ}$ in this experiment. The resultant spectra are measured by the FBG spectrum analyzer as shown in Fig. 12.

As shown in (3), when the slant angle of the fiber end is equal to or larger than $8^{\circ}$, the reflectance is equal to or less than $-98.74 \mathrm{~dB}$, and the return noise caused by the fiber end interface is much lower than that caused by the optical components before Position 4. Therefore, the return noise of the system increases inconspicuously with an increase in the number of the FBGs, and there is no significant deterioration of the SNR.
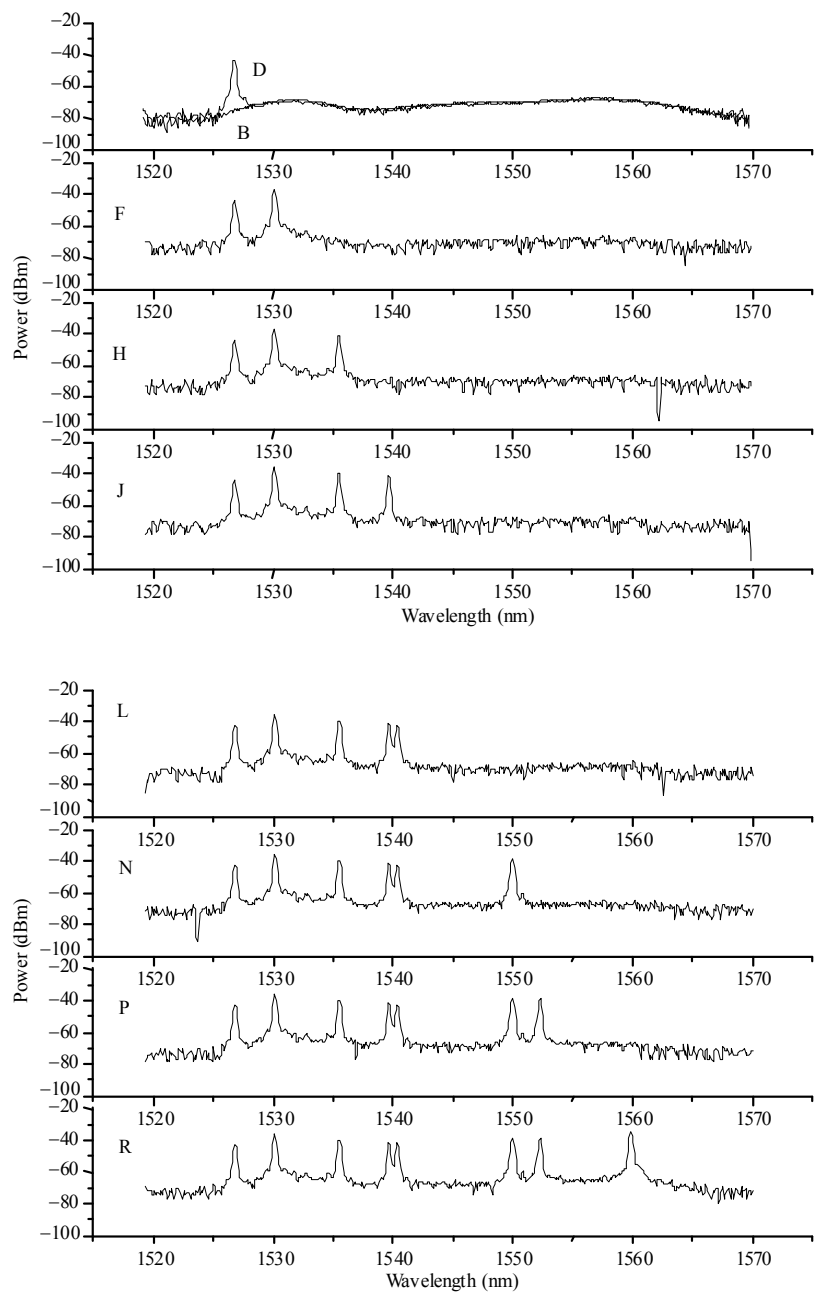

Fig. 12 Spectra of the return noise detected by the FBG spectrum analyzer when the fibers at Position 4 are bent (Curve B) and when each jumper together with the FBG is connected to each original port of the splitter one by one (Curves D-R).

\subsection{Contrast of the SNR of the parallel connection and serial connection}

In Section 4.2, we investigated the factors that affected the SNR in the FBG sensing system with the parallel connection. However, for practical applications, the serial connection is usually preferred due to the low power requirement of the light source. In the following section, we keep the same light power as shown in the above sections and compare the difference of the SNR between two setups.

In Fig. 13, Curves B and D depict the reflection spectra of the serial and parallel setups of the FBG, respectively. The calculated values of the SNR for the serial and parallel setups are approximately the same. This attributes to that the return noise increases with an increase in the signal.
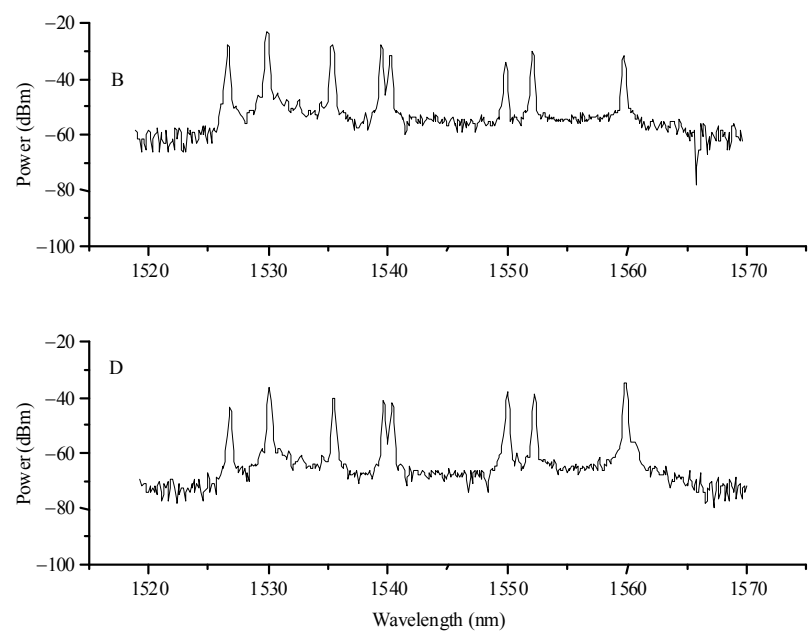

Fig. 13 Comparison of the SNR in the FBG sensing system with the parallel and serial setups.

In the serial FBG setup, the reflected signal becomes weaker when the FBG is closer to the end of optical path because of the insertion loss of the connectors between FBGs. At the same time, the return loss of the system increases due to the extra connectors.

Based on our analyses, the serial FBG connection is preferable so as to increase the signal power of the FBG sensing system. Besides, thermo-weld method is suggested to be used for the 
serial FBG setup to provide a low insertion loss [19]. By combining the two aspects, it is hoped that an FBG sensing system can provide us with the high signal power and high SNR.

\section{Conclusions}

The source of the noise as well as the signal attenuation of the FBG sensing system has been theoretically analyzed and experimentally tested. It is found that the optical noise caused by the ORL is the main source of noises in the system, and the coupler is the main source of the attenuation of the signal. Moreover, the cause of the ORL in fiber-optic elements is presented. Based on this, the optimization of the fiber optical sensing network in order to improve the SNR has been completed. Methods to suppress the noise caused by the fiber end interfaces of FBGs, including using index-matching fluid, bending fiber pigtails in the way mentioned in this paper and cleaving the slant angle of the fiber interfaces to be $8^{\circ}$, all contribute to the optimized SNR. Besides, thermo-weld method is suggested to be used for both parallel and serial FBG setups to provide a low insertion loss. The results would be a useful engineering tool to design the high SNR optical sensing system.

\section{Acknowledgment}

This work was supported by Natural Science Foundation of China (60977058), Science Fund for Distinguished Young Scholars of Shandong Province of China (JQ200819), Promotive Research Fund for Excellent Young and Middle-aged Scientists of Shandong Province (BS2010DX028) and Independent Innovation Foundation of Shandong University (IIFSDU2010JC002).

Open Access: This article is distributed under the terms of the Creative Commons Attribution License which permits any use, distribution, and reproduction in any medium, provided the original author(s) and source are credited.

\section{References}

[1] E. A. Mendoza, Y. Esterkin, C. Kempen, and Z. Sun, "Multi-channel monolithic integrated optic fiber Bragg grating sensor interrogator," Photonic Sensors, vol. 1, no. 3, pp. 281-288, 2011.

[2] G. E. Obarski and J. D. Splett, "Transfer standard for the spectral density of relative intensity noise of optical fiber sources near $1550 \mathrm{~nm}$," JOSA B, vol. 18, no. 6, pp. 750-761, 2001.

[3] Y. H. Chen, Y. T. Fu, and X. M. Shen, "Stray radiaton analysis caused by interior heat radiation in infrared optical system," Optica l Technique, vol. 32(z1), pp. 73-79, 2006.

[4] P. C. Noutsios, "Optical return loss measurements and simulation of an arbitrary array of concatenated reflective elements on field-installed optical links," Journal of Lightwave Technology, vol. 24, no. 4, pp. 1697-1702, 2006.

[5] M. Nakazawa, "Rayleigh backscattering theory for single-mode optical fibers," JOSA, vol. 73, no. 9, pp. 1175-1180, 1983.

[6] W. J. Dai, H. Y. Zhang, and Y. Q. He, "Estimation of optical signal-to-noise ratio and system design in all-optical network," Chinese Journal of Lasers, vol. 30, no. 12, pp. 1096-1098, 2003 (in Chinese).

[7] D. Marcuse, Light transmission optics. New York: Van Nostrand Reinhold Company, 1982.

[8] S. Y. Li, X. D. Tan, D. H. Huang, X. W. Yang, and H. M. Hu, "Analysis of reflection mechanism of ROSA with tilted fiber stub end face," Study on Optical Communications, vol. 32, no. 6, pp. 58-60, 2006.

[9] S. Chen and Y. Feng, "Effect of inclined surface of fiber end on high power fiber amplifier," Chinese Journal of Quantum Electronics, vol. 25, no. 6, pp. 686-691, 2008 (in Chinese).

[10] W. A. Gambling, H. Matsumura, and C. M. Ragdale, "Curvature and micro-bending losses in single-mode optical fibers," Optical and Quantum Electronics, vol. 11, no. 1, pp. 43-59, 1979.

[11] C. L. Xi, "A novel erbium-doped fiber broadband light source of ring from implement using double pumping," Optical Communication Technology, vol. 32, no. 12, pp. 14-16, 2008.

[12] J. Yang, T. H. Zhang, H. Z. Yang, Y. Z. Lu, C. P. Zhang, Y. Y. Sun, and X. L. Xia, "Research on return wave interference of X-type optical fiber coupler," Journal of Optoelectronics Laser, vol. 14, no. 9, pp. 933-935, 2003. 
[13] J. M. Whitney, K. Takami, S. T. Sanders, and Y. Okura, "Design of system for rugged, low-noise fiber-optic access to high-temperature, high-pressure environments," Sensors Journal, vol. 11, no. 12, pp. 3295-3302, 2011.

[14] S. W. Lloyd, V. Dangui, M. J. F. Digonnet, S. H. Fan, and G. S. Kino, "Measurement of reduced backscattering noise in laser-driven fiber optic gyroscopes," Optics Letters, vol. 35, no. 2, pp. 121-123, 2010.

[15] Y. Y. Xie, M. Nikdast, J. Xu, W. Zhang, Q. Li, X. W. Wu, Y. Y. Ye, X. Wang, and W. C. Liu, "Crosstalk noise and bit error rate analysis for optical network-on-chip," Study on Optical Communications, vol. 32, no. 6, pp. 58-60, 2006.

[16] Y. K. Xu, "Research on light amplification panel based on stimulated radiation," Materials Science Forum, vol. 663-665, no. 344, pp. 344-347, 2010.

[17] H. T. Nguyen, C. Fortier, J. Fatome, G. Aubin, and J. L. Oudar, "A passive all-optical device for $2 \mathrm{r}$ regeneration based on the cascade of two high-speed saturable absorbers," Journal of Lightwave Technology, vol. 29, no. 9, pp. 1319-1325, 2011.

[18] R. S. Luis, A. Teixeira, and P. Monteiro, "Optical signal-to-noise ratio estimation using reference asynchronous histograms," Journal of Lightwave Technology, vol. 27, no. 6, pp. 731-743, 2009.

[19] H. Q. Li, Y. Y. Huang, T. H. Li, and H. L. Song, "Research on the inserting loss of fiber connector with high power," Optical Communication Technology, vol. 34, no. 2, pp. 46-48, 2010 (in Chinese). 